\title{
Do Distracted Driving Laws Impact Cell Phone Usage on (Preventable) Outcomes While Driving: A Systematic Review of the Last 20 Years?
}

\author{
Nudar Bhuiya ${ }^{1}$, Lisa Hollister ${ }^{2}$, Thein Zhu \\ ${ }^{1}$ Indiana University School of Medicine; ${ }^{2}$ Parkview Trauma Centers, Fort Wayne, IN
}

\begin{abstract}
Background/Objective:
Distracted driving due to cellphone use can lead to serious and fatal consequences. In 2017, an estimated $14 \%$ of all distraction-affected fatal crashes in the U.S. were related to the driver's cellphone use. Many states have passed distracted driving laws to help reduce the number of unfortunate outcomes related to a driver's cellphone use. It is important to evaluate whether these laws are truly effective at building safer roads. The main objective of this proposed study is to execute a systematic review of literature published in the last 20 years in order to further the understanding of how distracted driving laws affect drivers' cellphone usage and its associated outcomes.
\end{abstract}

\section{Proposed Methods:}

A systematic review will be conducted using the Preferred Reporting Items for Systematic Review and Meta-Analysis (PRISMA) statement established by Moher et al. The various steps that will be taken to properly select and analyze articles include eligibility criteria, information sources and search strategy, study selection, evaluation of selected articles, data collection, and summary measures.

\section{Expected Results:}

After implementing the various steps from the PRISMA statement, certain articles will be selected to fulfill the research objective. It is expected that most of the publications selected for this systematic review will be in support of distracted driving laws. It is also expected that these laws are in fact effective at reducing drivers' cellphone use and various negative outcomes, such as crashes.

\section{Conclusions/Potential Impact:}

A systematic review will be completed utilizing the proposed protocol written in the summer of 2020. If needed, additional details and changes will be applied to the protocol before the study officially begins. This systematic review may help us gain an understanding of the effects of distracted driving laws, which may impact the approach to creating future policies and interventions intended to promote safer driving roads. 